\title{
Advanced system concepts for future civil aircraft-an overview of avionic architectures
}

\author{
A Nadesakumar and $\mathbf{R}$ M Crowder \\ Department of Electrical Engineering, University of Southampton \\ C J Harris \\ Department of Aeronautics and Astronautics, University of Southampton
}

\begin{abstract}
The avionics systems of state-of-the-art commercial aircraft have become increasingly complex and sophisticated, in order to meet the ever increasing performance and reliability requirements. With the capability of the avionics technology improving by an order of magnitude every few years, it is envisaged that the current philosophy of one box-per-function will soon reach its limits in terms of cost, finctionality, reliability, and certification. The proposed solution is the integrated systems configuration, using distributed processing, where the computational resources are shared between many functions, therefore improving the reliability, availability, survivability, and extensibility of the overall system. Futhermore, this approach will also provide the potential for reducing the acquisition, maintenance and operating costs. The paper discusses the limitations of the current avionic system's architecture in dealing with the high levels of functionality required by the state-of-the-art aircraft, and discusses the philosophy of the integrated modular avionics, which represents a change in philosophy of avionics design to a decentralized, distributed architecture that allows interchangeable components within a distributed aircraft avionic system. The paper also addresses a number of specific issues considered necessary far the implementation of a decentralized, distributed architecture such as data bus requirements, electromagnetic and radio frequency prevention, and fault tolerance But it also argues that for true systems integration, a new culture is required based on open systems with a set of inherent quality features such as conformance, robustness, extendibility, compatibility, and reusability built into the architecture.
\end{abstract}

Key words: interoperability, avionics architecture, integrated modular architectures, fault tolerance

\section{INTRODUCTION}

The avionics systems in commercial aircraft are organized into sub-systems for functional areas such as flight control, engine control, navigation, communication etc. These sub-systems have their own dedicated computers ('black boxes'), housed in the avionics bay as line replaceable units (LRUs), where they are provided with power and conditioned air as required. Communication between the various computers in the avionics bay is achieved through data buses-the ARINC 429, while communication between the peripheral devices, that is, sensors, actuators, pumps, valves, and the computers which control them is achieved via dedicated point-to-point data links. To satisfy airworthiness requirements, critical components and data channels are replicated. This represents the basic architecture of the avionics system as it exists today in modern commercial aircraft.

The architecture, however, suffers from a number of problems. One of the main problems is the large number of wires and interconnections found in the system.

Another problem is the use of a variety of computers to perform each function. Although LRUs have eased on-line maintenance through removal and replacement procedures, an extensive supply of spares and a wide range of expensive test equipment with skilled maintenance personnel to operate them has to be maintained at various points along the airline's routes (1). This imposes substantial operating overheads on the airline operator.

A further disadvantage of the current avionic architecture is the lack of flexibility to accommodate updat-

The MS was received on 29 December 1993 and was accepted for publication on 10 September 1994. ing the system extensions. Since the architecture is inherently coupled, that is, there is a close relationship between all of the system elements, any change to one element of the system has an effect on another-such modifications make system upgrades expensive and time consuming.

In view of this the avionics community (that is, the airframe manufacturers, the airline operator and the equipment manufacturers) have argued for a new approach to avionics. For a number of years the avionics community at large have called for $(2-4)$ :

(a) a reduction in overall cost of ownership through reduced spares requirement and equipment removal rate;

(b) a reduction in weight and volume of wiring leading to increased range and payload;

(c) improved built-in-test (BIT) coverage to provide better maintenance diagnostics, improved fault detection, and reduced unconfirmed removals;

(d) maintenance-free dispatch to achieve quick turnaround times;

(e) resource sharing to reduce LRU count;

(f) standardization at the functional interface to provide hardware and software interoperability (that is, vendor/product independence).

In order to achieve these goals, it is clear that the avionic system configuration must move away from the traditional approach of point-to-point, one box-perfunction philosophy. The concept which has been suggested is the modular, integrated systems approach where the computer resources are shared by different functions. The idea is that resource sharing would minimize functional replication in hardware and software providing cost savings, with the modularity allowing flexible growth. 


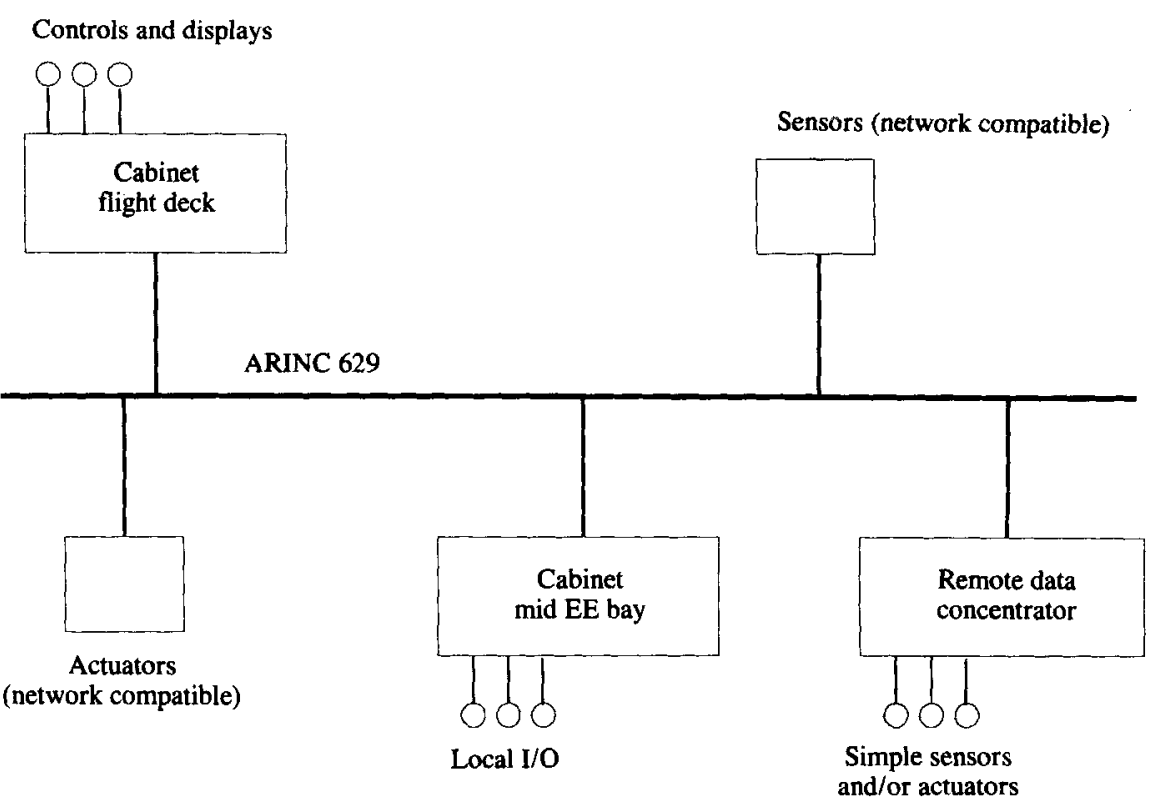

Fig. 1 Integrated modular avionics-system overview

The concept of functional integration is, however, not new-it was considered as early as 25 years ago (5). Although numerous attempts were made in implementing a fully integrated avionic suite, none were practical until recently, due to limitations of enabling technology and cost effectiveness. With recent advances in technology, integrated architectures which were once not technologically feasible and cost effective have now become realizable. To this end, a number of avionics research and development organizations have proposed a number of avionic system architectures-from a centralized architecture to a fully distributed one.

This paper presents an overview of the issues concerned with the introduction of new avionic architectures, that will provide a solution to the demands of both system integration and operation. While aircraft systems such as actuators and sensors form an integral part of the control structure, this paper will focus on a number of the major architectural issues.

\section{INTEGRATED MODULAR AVIONICS (IMA) ARCHITECTURE}

One of the most promising architectures to emerge as a result of research and development activity is the integrated modular avionics (IMA) architecture (4-6). The architecture takes advantage of recent developments in microprocessor design, namely high data throughput and inherent fault tolerance. It makes use of powerful computers for the processing of applications software. These computers together with hardware modules are housed in a cabinet forming a subsystem with a common chassis design, common fault tolerant processing, redundant power supplies and flexible aircraft interfaces. Several of these cabinets are interconnected by ARINC 629* data buses which also connect avionic hardware and other peripherals (that is, sensors, actu-

\footnotetext{
* ARINC (Aeronautical Radio Incorporated) is a corporation in which the United States scheduled airlines are the major shareholders. Other shareholders include aircraft manufacturers, air transport companies and foreign flag carriers. The ARINC 629 is the new, bi-dimensional, high speed ( $2 \mathrm{Mbits} / \mathrm{s})$ data bus specifically designed to carry digital data between avionics equipment on commercial aircraft.
}

ators, indicators etc.) outside the cabinet to form an integrated system for performing all the avionic functions on the aircraft, see Fig. 1. In this approach sensors and actuators are treated in the same way on any plug-in unit, only the input-output functionality is different.

A concern with this kind of system is that since transfer of data takes place in a more shared manner, a centralized failure in the architecture can bring about simultaneous loss of functions utilizing shared resources. To overcome this, fault tolerance is applied in IMA to increase resource availability and integrity. In IMA fault tolerance is achieved through redundancy and high integrity monitoring.

Although fault tolerance is required to meet availability and integrity requirement goals in IMA, it also satisfies the airline goal for deferred maintenance. Furthermore, high integrity monitoring also satisfies the airlines' desire for improved fault isolation, better maintenance diagnostics and reduced unconfirmed removal rates. Reports indicate that a fully integrated IMA architecture would reduce the number of unconfirmed removals by as much as six times over today's line replaceable unit based avionics (4).

The resource redundancy required to extend the mean-time between maintenance alert/action (MTBMA) is dependent upon the length of the extended maintenance interval and the statistical probability of successfully completing that interval before total equipment failure (2). The desired extended maintenance interval is usually dictated by individual airline philosophy. However, as guidance, IMA has established for a fully fault tolerant suite, a reliability requirement (or MTBMA) of 15000 hours to first alert, which must not cause loss of function, and 99 per cent probability providing full function for another 200 hours, that is, capability of getting the aircraft to base for maintenance and repair (2). These requirements are considered to be adequate to eliminate unscheduled removals of IMA common components.

It is envisaged that the adoption of IMA architecture will increase the acquisition cost, that is, development 
and production costs. However, the increase in initial acquisition cost is expected to be offset by significant reductions in ownership cost, that is, through life operating and support costs, and in technical reports and updates in equipment.

The acquisition cost is expected to be dominated by the development costs of the application software and the software associated with the implementation of the architecture. But it should be noted that software development costs dominate virtually all current avionics project costs. However, in IMA, potential reductions in software development cost can be expected through software modularity, re-usability and portability. Indeed if object oriented system design is incorporated additional software quality attributes are assured.

The incorporation of redundancy, enabling deferred maintenance, for flight safety is also expected to add to the acquisition cost; this of course already applies to existing avionic systems. However, reductions in acquisition cost can be expected through competition. The basic IMA concept allows the utilization of common and standard flight hardware. This provides the equipment manufacture with increased marketing opportunities fostering competition-a potential factor in reducing acquisition cost. Reductions in development cost can also be expected through resource sharing, since the IMA concept allows common resources to be shared between different avionic functions, the functional duplication of hardware and software elements found in current avionic systems is kept to a minimum-frequently only associated with multiversions for safety critical areas.

The ownership cost, in general, is dominated by the maintenance and support costs. However, the adoption of IMA is expected to result in a significant reduction in ownership cost. The fault tolerant architecture provides fail operational capability through any first failure and allows maintenance to be deferred until a convenient time. The advantages derived from deferred or scheduled maintenance are many.

Deferred maintenance reduces the number of unscheduled maintenance breaks and avoids extremely costly flight delays and cancellations. It also has implications on manning levels and provisions of spares. Deferred maintenance provides the potential for reductions in intermediate shop-level maintenance providing cost savings on maintenance personnel and sig- nificant savings on test equipment. It also reduces the number of spares needed to be kept on the airfield reducing the cost of spares inventory.

The high integrity monitoring inherent in the architecture provides improved diagnostics capability. This should reduce the number of unconfirmed removals. A line replaceable module (LRM) found faulty can be replaced from the 'small' stock held at the airfield, lowering the flight line spares avionics inventory and maintenance personnel. The cost incurred in unconfirmed removals is attributed to the time taken to test and return good boxes for servicing and the cost of necessary spares to support this operation. Reports indicate that a dominant contributor to the cost of maintenance is unconfirmed removals (2). Improved diagnostics would also allow swift flight maintenance action which results in higher dispatch availability.

The adoption of IMA architecture is also expected to offer significant savings in weight and volume. Weight savings stem from the use of the ARINC 629 data bus and the 'avionic cabinets'. The utilization of the bidirectional ARINC 629 data bus results in a lower wire count providing substantial reduction in aircraft wiring, weight and build time, with consequent significant savings in production cost and concomitant improvement in reliability. Boeing expect a wire weight saving of about $522 \mathrm{~kg}$ (five additional passengers) on a 757 sized aircraft (7).

The weight of the cabinet modules (LRMs) are dependent on the manufacturer's implementation and thus are difficult to specify. However, weight savings can be expected from the use of cabinets. Since each cabinet is capable of providing multiple avionic functions, a single cabinet is able to replace a number of aircraft functions, lowering the weight and volume of the avionics on the aircraft. Initial architectural studies carried out by Boeing indicate that for typical avionics and electrical system functions, about 50 individual LRMs can be integrated in eight integrated avionic computer system (ICAS) cabinets with an estimated saving of 25 per cent in recurring costs, 30 per cent reduction in weight, 46 per cent reduction in volume and 84 per cent reduction in the number of individual power supplies, (8).

The advantages to be gained from implementing the aircraft avionics using the IMA concept are outlined in Table 1.

In summary, the IMA architecture is envisaged to

Table 1 Benefits of integrated modular avionics

\begin{tabular}{|c|c|}
\hline Aspects & Comments \\
\hline $\begin{array}{l}\text { Network system with shared } \\
\text { resources }\end{array}$ & $\begin{array}{l}\text { - Reduces aircraft wiring, weight and build time } \\
\text { - Decouples hardware from software: } \\
\text { flexible to accommodate hardware and software upgrades }\end{array}$ \\
\hline $\begin{array}{l}\text { Cabinet with multifunctional } \\
\text { processing capability }\end{array}$ & $\begin{array}{l}\text { - Single cabinet is capable of replacing many individual aircraft functions: } \\
\text { reduces functional duplication of hardware and software; } \\
\text { reduces weight and volume of avionic equipment }\end{array}$ \\
\hline Fault tolerance & $\begin{array}{l}\text { - Increased reliability and maintainability } \\
\text { - Deferred maintenance: } \\
\text { reduces number of unscheduled maintenance; } \\
\text { reduces intermediate shop level maintenance: } \\
\text { savings on maintenance personnel } \\
\text { savings on test equipment } \\
\text { reduction in spares }\end{array}$ \\
\hline Multi-vendor support & - Customer not locked to one vendor \\
\hline Flexibility of equipment location & - Optimizes equipment space \\
\hline Compatible with fibre optics & - Provides EMI/RFI immunity \\
\hline
\end{tabular}


provide benefits to the airframe manufacturer, the operator and the equipment manufacturer. The benefits to the airframe manufacturer include:

(a) reduced avionics cost, weight and volume;

(b) reduced manufacturing and development cost;

(c) more flexible architecture to accommodate technological upgrades and airline-specific avionic installation.

For the operators, a significant reduction in operating cost is envisaged through better maintenance. Other benefits to the operator include:

(a) the ability to defer maintenance;

(b) no maintenance required during turn-rounds;

(c) improved fault isolation;

(d) reduction in number of no fault found removals;

(e) reduced spares requirement.

For the equipment manufacturer, the architecture allows:

(a) increased marketing opportunity;

(b) easier to offer system upgrades and additional systems.

A major concern of IMA architecture is certification. Since functions with different criticality are processed in one cabinet there is always the potential for undesirable interaction between separate avionic functions. As a result questions have been raised regarding the certification of the IMA concept, particularly with respect to common mode failures, partitioning integrity, and onboard software loading. Boeing have had preliminary discussions with the Federal Aviation Authority (FAA) on this matter, and envisage no formidable problems, provided that strict fault tolerance and robust hardware and software partitioning are adhered to.

In recent years the IMA concept has been extensively discussed in the Systems Architecture and Interface (SAI) subcommittee of the AEEC (Airline Electronic Engineering Committee) and their recommendations have been well documented in ARINC Project Paper 651 (2). Although more recently, the concept has been finally approved by the AEEC, the early IMA systems are likely to only involve a limited number of systems, so as to contain the development risks associated with the new concept. This is exemplified by considering the Boeing 777 aircraft, the first to implement ARINC 651, in which the airplane information and management system (AIMS) is designed based on the IMA concept. In the AIMS architecture the conventional LRUs, which typically contain a single function, are replaced with dual integrated cabinets which provide the processing, power supply, the $\mathrm{I} / \mathrm{O}$ hardware and software to perform several functions $(4,9,10)$.

\section{RELATED ISSUES}

\subsection{Data bus}

In order to realize the full potential of an integrated system like the IMA architecture, a highly efficient, advanced data bus is required. The ARINC 429 data bus, although suitable for use in simple aircraft is considered inefficient for use in today's complex avionic systems. Comparison of data in commercial avionics used today would indicate that in order for a data bus to serve a modern aircraft it must be bi-directional and highly reliable with sufficient data rate to accommodate the integration of the entire avionic suite on the aircraft. Futhermore, it should not rely on a bus controller, and be flexible enough to accommodate changes. In summary, for a data bus to serve the next generation of commercial aircraft, it should have the following qualities $(11,12,13,14)$ :

(a) the bus must be bi-directional to achieve minimum wire count;

(b) bus controllers must be avoided from the single point failure and system inflexibility point of view;

(c) the data rate should be sufficient (that is, highspeed) to handle the entire cockpit integration, including radar and other applications such as the digitizing and multiplexing of inherently high bandwidth data, for example, audio and video;

(d) the data specification format, that is, labels, should be broad enough to cover the range of equipment that is expected in the next $10-15$ years due to advances in technology;

(e) the bus should be flexible to accommodate future capability upgrades and airline specific avionic installation;

(f) the bus should allow the use of reasonably priced commercial hardware for input and output interface.

\subsection{Electromagnetic and radio frequency prevention}

One of the concerns of employing data buses to effect communication is its susceptibility to high intensity electromagnetic and radio frequency interference (EMI and RFI). This concern has been heightened by two recent trends in technology: the increased use of composite material on aircraft for both aircraft skin and primary structure, and the application of advanced microelectronics technology in aircraft.

At present aircraft avionic systems and their associated data channels are protected from EMI and RFI sources by the Faraday shielding inherently provided by the aircraft skin. Composite materials, however, do not offer shielding to external EMI and RFI sources, and thus require additional shielding for the data channels, which incur undue weight and cost penalty. Although it may be relatively easy to provide adequate shielding, the verification procedures to test their effectiveness become an expensive and time consuming operation.

Fibre optics offer an ideal solution to the problem by eliminating the need for the heavy electromagnetic shielding of the cable, and by eliminating most of the devices usually needed for protecting the electronic circuit from conducted electromagnetic interference. Although extensively used in the telecommunication industry, several factors limit the application of fibre optics on aircraft.

At present the main factors hindering the near time acceptance of fibre optic systems on aircraft appear to be the cost effectiveness of the overall system and the instability, maintainability and in-service reliability of the connectors in the aircraft environment. This does not mean, however, that fibre optics have not been used on commercial aircraft. Early examples of fibre optics 


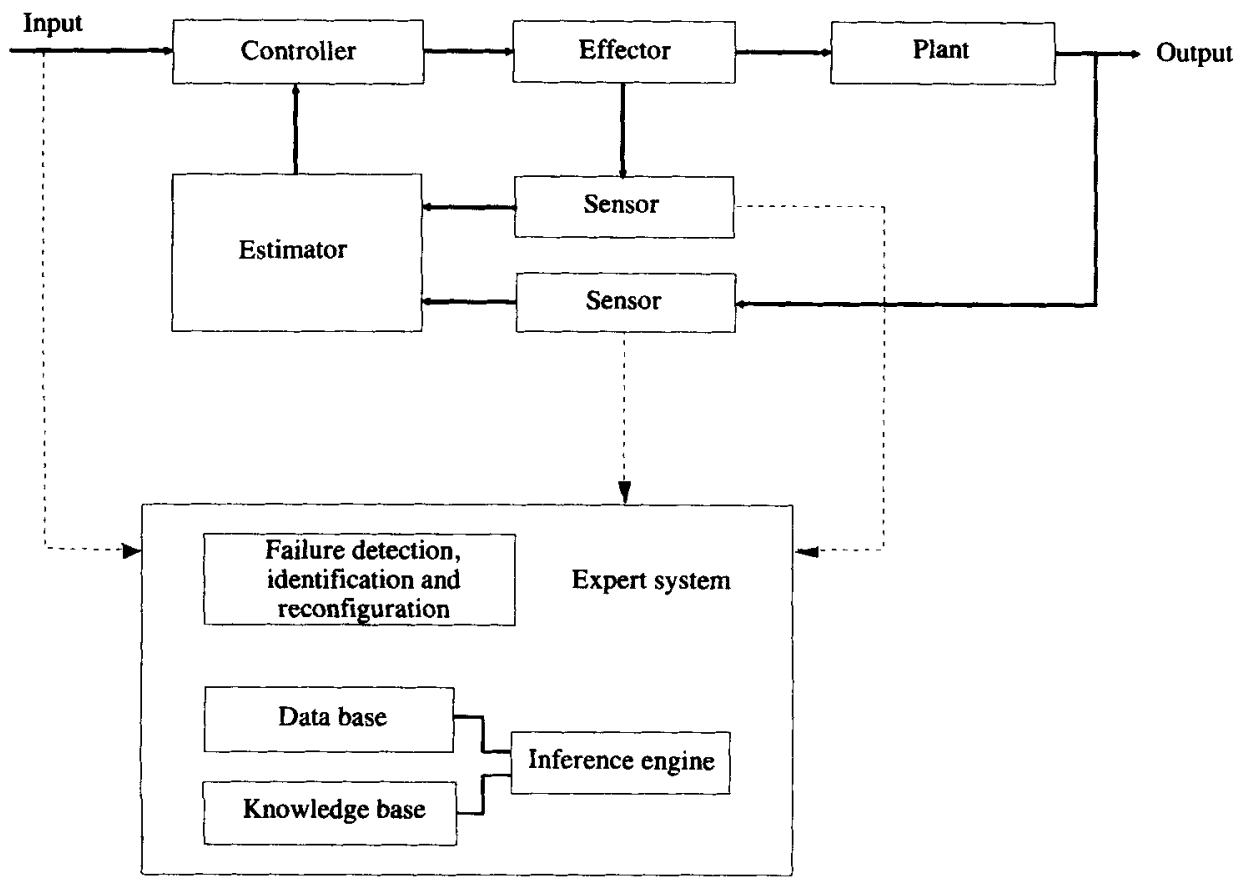

Fig. 2 Expert system approach to analytical redundancy

on commercial aircraft for non-flight critical applications include the Boeing 747 in-flight entertainment (IFE) system; the reliability of these systems was poor, though efforts are being made to improve on it.

Although some fibre optic component technologies are mature, such as optical fibres; others such as connectors and couplers still need further development before the full performance potential of the system can be realized. In order for fibre optics to become truly competitive with metallic cables and be readily accepted for use on aircraft:

(a) more robust and reliable connectors are needed;

(b) ways need to be found to locate faults more easily;

(c) simple, easy to use, test equipment and procedures are necessary.

\subsection{Fault tolerance}

Fault tolerance has always been an essential requirement of aircraft design, and is also one of the key components in achieving a high level of integration. The technique currently adopted in aircraft to provide fault tolerance is based on the concept of hardware redundancy, that is, the use of multiple processors, communication paths, sensors and actuators accompanied by a voting system. Although the concept has provided useful information for fault detection, isolation and accommodation the benefits have been achieved at a price: increase in weight and cost.

Modern approaches to fault tolerance (that is, analytical redundancy and knowledge-based concepts) provide the potential for eliminating the hardware redundancy, while still maintaining the high level of reliability and availability. These concepts make use of efficient fault detection, identification and accommodation (FDIA) strategies. On aircraft, the concepts can be implemented on the on-board computers providing savings in weight, volume and maintenance. Hardware redundancy, however, cannot be completely eliminated because it is essential for reconfiguration. Therefore the application of the said concepts on aircraft is seen as reducing the level of hardware redundancy (for example, from a triplex system to a duplex system).

Fault tolerance using analytical redundancy is based on the generation of residuals, and there are a number of techniques (for example, dedicated observers, fault detection filters) which can be used to generate this signal $(14-16,17)$. One of the problems associated with the use of analytical redundancy for FDI application has been the sensitivity of the detection system to modelling errors $(14,18)$. Since the system model on which the redundancy is based is not exactly known, there is a possibility that the actual system outputs may not match the model outputs, even when there are no faults. Thus the residuals will not be zero in general and some form of threshold would have to be used to distinguish faults. But the problem with using thresholds is that they reduce the sensitivity of the detection system, choosing a threshold too low increases the rate of false alarms and choosing it too high reduces the net effect of fault detection. This problem has, however, been recognized, and several schemes have been proposed in recent years to increase the robustness of the fault detection system. These include robust observer schemes, and a proper choice of the threshold and adaptive threshold $(\mathbf{1 7}, \mathbf{1 9}, \mathbf{2 0})$.

Recently, Merrill and co-workers from NASA Lewis Research Centre carried out a real time evaluation study to detect sensor failure using analytical redundancy. Their studies indicate that software based failure detection algorithms do indeed work and work quite well. Furthermore, their studies indicate that the algorithms can be implemented in a realistic computer environment with an update rate consistent with real time operation (21).

Fault detection and identification (FDI) systems using analytical redundancy concepts are designed based on the assumption that a good mathematical 
model of the system being monitored is available. As a result the achievable quality of the system depends on the quality of the model. For large complex systems it may not always be possible to obtain a mathematical model of the system and this is one of the limitations of this approach.

When analytical models are not available for the task of FDI, knowledge-based models, for example expert systems, can be used instead, Fig. 2. This is one of the advantages of knowledge-based systems. A further advantage is that they provide the facility to represent experiential knowledge which is hard to come by and difficult to capture numerically. Expert systems can not only be used to complement analytical redundancy and provide failure diagnosis, but they also provide the capability to predict faults (that is, prognostics) before they occur. The implementaion of failure prediction on an aircraft diagnostic system would provide several benefits:

(a) rapid fault isolation-additional information is made available for the identification of faulty elements;

(b) the ability to plan maintenance action;

(c) reduction in the probability of 'knock on' faults, and enhancement in safety.

Both analytical redundancy and knowledge-based systems have their limitations (for example, the system may be too complex for mathematical modelling; the completeness of the knowledge base cannot be guaranteed; there may be an excessive number of rules). But the limitations can be overcome by combining the two schemes to yield a flexible and efficient diagnostic system-the intelligent quantitative and qualitative (IQ ${ }^{2}$ ) system (Fig. 3), however, $\mathrm{IQ}^{2}$ schemes may incur a significant increase in computational expenditure. But recent work on neurofuzzy algorithms allow both symbolic and numeric information to be considered within a unified mathematical framework without any additional overheads (22).

Although large increases in processing speed are becoming available with the development of high throughput 32 bit processors, it is envisaged that the computational requirements (greater than ten-fold) needed to implement an expert diagnosis system for all of the aircraft subsystems are beyond current avionic computational capability $(23,24)$. A solution to this problem would be to use parallel computer architecture, that is, parallel processing. With the advent of transputers, recently, it has been shown that fault tolerant systems can be built based on parallel processors. Systems designed on this concept are said to offer not only high reliability and high performance but also lower cost, graceful degradation and enhanced system maintainability (25). As with all new technology the problem of certification needs to be addressed, however this is outside the scope of this paper. But current research at the University of Southampton Parallel Applications Centre on full authority engine digital controls, indicates that the above methodology is both feasible and certifiable. Another major concern is the software reliability. Since both the concepts are software based, reliability of software is paramount. $\mathrm{N}$-version programming has been suggested as a solution to the software reliability problem.

\section{DISCUSSION}

In assessing any candidate avionic architectures the qualities or attributes sought should be identified and evaluated. Current and future aircraft airframes are likely to have in-service times of in excess of 30 years, yet much of the information-based technology used in

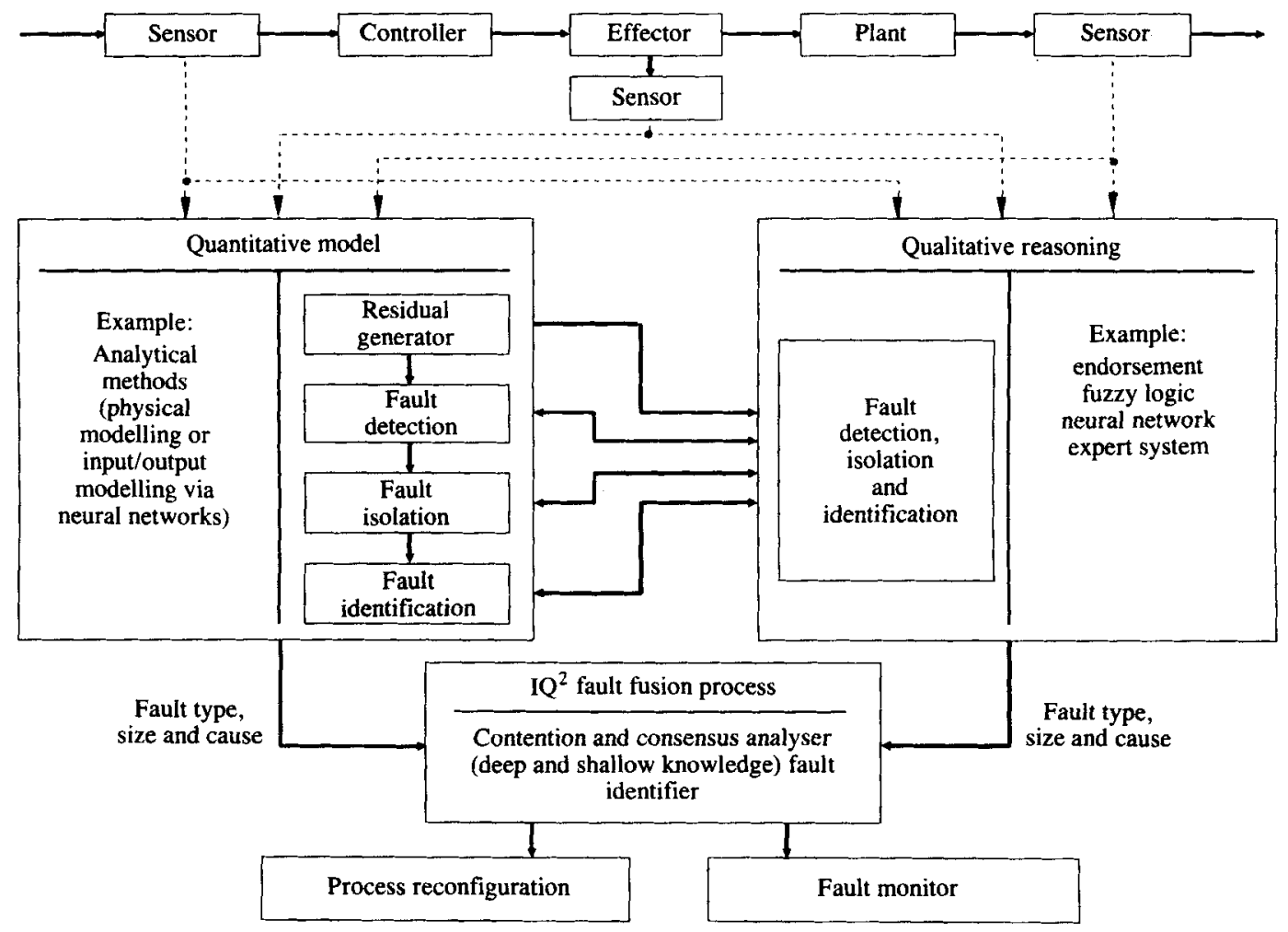

Fig. 3 The $\mathrm{IQ}^{2}$ system 
avionics has an ever decreasing technical half-life (typically 5-7 years), leading to rapid (but unpredictable) technical obsolescence. The conflict between longer air frame life and shorter subsystems life demands that future architectures have an open systems (see OSI in computer communications) design philosophy (26).

Open systems in this context means the ability to run a range of varying application-specific subsystems across heterogeneous platforms under different operating conditions, modes, standards etc. in a transparent and cohesive manner. Open systems conceptually implies inter-operation of components (avionic subsystems), portability of components and applications across heterogeneous platforms (including operating systems) and the ease of integration of disparate/ separate components into a coherent system design. It represents a change in philosophy of avionics design to a decentralized, distributed architecture that allows design of interchangeable avionic components within a distributed aircraft avionic system. This approach requires a set of principles to be defined:

1. Composability is the ease of construction of total systems from structured connections of components (subsystems).

2. Transparency is the independence of the interoperation of two components from their physical locations and implementations.

3. Extensibility is the ease of extension of a total system by extension to existing components or addition of new components.

4. Scalability is the evolution of a system from a small to large scale, representing increasingly complex abstractions, interconnections, requirements, etc. without a concomitant increase in design and implementation cost.

5. Portability is the ease of implementation of a system and subsystem on multiple physical platforms.

6. Compatibility is the ease with which total system components can be replaced or interchanged.

7. Interoperability is the ease with which total system components can communicate and interoperate to provide total system level functionality.

Scalability is only possible once the system is open to extension, this extensibility however, requires that new components can seamlessly interoperate with existing subsystems, and with other new components integrated with the total system. Interoperability is the critical capability within any open system and has been the 'holy grail' of military command and control systems $(26,27)$, and is believed to be the critical feature of any future aircraft avionics architecture.

The achievement of true interoperability within the avionics industry requires a change in culture of design and project management. What is essentially required is a change to product culture, where progress is measured not in terms of the completion of individual projects/ systems, but in terms of the number and quality of reusable components and subsystems developed. The highly desirable attribute of this culture is the associated development of portable generic subsystems technologies which may be readily extended and transferred from product to product. Certainly ARINC 651 offers the potential of partially fulfilling the change in culture.
The achievement of interoperability, together with the need for provable, correct and robust system behaviour, constitutes a manifesto for inherent quality in embedded avionic systems. Quality factors sought within this philosophy are:

1. Correctness or conformance: the ability of the system to exactly perform its tasks.

2. Robustness: the ability of the system to function even under abnormal or unanticipated conditions.

3. Extensibility: the ease with which the system may be adapted to changes in specification.

4. Compatibility: the ease with which system elements may be combined with each other into a coherent system.

5. Re-usability: the ability of the system elements to be used in whole or in part, in new system implementations.

The advantages in making the change to a quality product centred development culture in avionics must be great enough to overcome the short-term inertia of the project/specialized non-generic culture. On a project by project perspective it is often easier to ignore the longer term issues of re-use, compatibility and extensibility, for the sake of short-term expediency. The most significant driver in this direction is the need to reduce through-life costs of the system operator/owner. Reduction of through-life costs is dependent on providing an infrastructure or architecture and associated interface standards that inherently provide very low resistance to change or evolution. The ability to change existing sub-system components without impacting on other sub-system elements is an open systems requirement.

The quality factors of extensibility, re-useability and compatibility are essentially infrastructural issues that require a flexible, decentralized architectural design, composed of coherent modules or components connected by well defined interfaces. This ultimately leads to a component orientated perspective of future avionic systems construction, whereby an avionic system is a loosely coupled connection of components (hardware and software) communicating by open systems protocol to produce appropriate system behaviour, the realization of a given component should be transparent to the rest of the system, dependencies between components should be based on external, highly visible, standardized connections.

Within software engineering, a component-based approach to system synthesis is increasingly being utilized to tackle the problems associated with software complexity and distributed system-this is called object orientation or more generally object technology (28). It demonstrates that large-scale systems can be constructed with off the shelf components without notification, each contributing to total systems integration and functionality. The essence is the construction of a total system from packaged components integrated within an infrastructure that allows modules to be pluggable, but to adhere to this metaphor of open component systems integration requires a framework that provides a scheme for the systematic definition of structural composition, connectability and interrelationships of avionic sub-system components. This framework and associated standards for a truly component-based 
avionic systems require the evolution of a new reference model for component-based integration that inherently features all the quality attributes discussed above. Interestingly, this has been achieved for mobile autonomous robotic systems operating in unstructured outdoor environments (29) and the same approach is being investigated for intelligent vehicle highway systems (IVHS) and Air Traffic Control, so why not avionics architectures?

\section{CONCLUSIONS}

This paper has discussed the limitations of the current avionic architectures when dealing with the high level of functionality required by advanced civil aircraft. The case has been presented that to achieve this requirement, avionic systems must move away from the traditional point-to-point, one function per box, to a decentralized, distributed architecture.

In the discussion the authors have presented a case for open systems within avionics, and defined the principles and benefits of this approach. The IMA architecture offers the potential for achieving this. The paper has detailed a number of areas that will present challenges to the implementation of integrated architectures, and has purposed approaches to their solution.

\section{ACKNOWLEDGEMENTS}

The authors would like to acknowledge the industrial members of the Collaborative Research Programme into Secondary Power Systems for their assistance, and the UK Department of Trade and Industry for the programme funding. The opinions expressed in this paper are entirely those of the authors and do not necessarily represent those of the programme members.

\section{REFERENCES}

1 Little, R. Advanced avionics for military needs. Comp. Cont. Engng $J$, IEE, January 1991, 29-34.

2 AEEC 1991. Design guidance for integrated modular avionics. ARINC Report 651, November, 1991.

3 Archer, H. S. Digital avionics for modern aircraft-a case study into the problems and promise of aircraft electronics. Proc. IEEE, $1985,144-157$

4 Morgan, M. J. Integrated modular avionics for next-generation commercial airplanes. NAECON '91, Dayton, Ohio, May 1991 pp. 9-12.

5 Janex, A. Reflections on avionic integration, AIAA Paper No. 883950-CP.

6 Subra, H., Paquier, M. and Graves, D. New avionics architecture concept for commercial aircraft. Seventeenth ICAS Congress, Stockholm, Sweden, Vol. 1, 1990, pp. 607-616.
7 Shaw, J. L. and Sutcliffe, P. L. ARINC 629 data bus system. Civil avionics-the future international scene, RAeS Proc., March 1988, 109-129.

8 Sutcliffe, P. L. Integrated avionics-future developments. Civil avionics-the future international scene, RAeS Proc., March 1988, 91-108.

9 Bailey, J. Honeywell joins 777. Flight International, 12-18 December 1990 , pp. $32-34$.

10 Hopkins, H. Delivered with feeling. Flight International, 20-26 November 1991, pp. 31-38.

11 Card, M. A. Evolution of the digital avionics bus. Proc. IEEE, 1983, 8.5.1-8.5.6.

12 McGough, J. Evaluation of data busses for flight critical control applications. Proc. IEEE, 1986, 718-727.

13 Stainslaw, D. L. General aviation data bus update. AIAA Paper No. 84-2637.

14 Frank, P. M. Fault diagnosis in dynamic systems using analytical redundancy and knowledge-based redundancy - a survey and some new results. Automatics, 26(3), 1990, pp. 454-474.

15 Labarrere, M. Aircraft sensor failure: detection by analytical redundancy. System and Control Encyclopedia, Vol. 1, pp. 246-252 (Pergamon Press, Oxford).

16 Patton, R. J. Fault detection and diagnosis in aerospace system using analytical redundancy. IEE Colloquium on Condition Monitoring and Fault Tolerance, Savoy Place, London, November 1990 , pp. $1 / 1-1 / 20$.

17 Patton, R. Fault diagnosis in dynamic systems: theory and application. (Eds R. Patton, P. Frank and R. Clark), 1989 (Prentice Hall).

$18 \mathrm{Xu}$, Y. M. Knowledge based fault monitoring for large complex systems. PhD Thesis, Department of Aeronautics and Astronautics, Southampton University, 1991.

19 Emami-Naeini, A., Akhter, M. M. and Rock, S. M. Effect of model uncertainty of failure detection: the threshold selector. IEEE Trans. on Automatic Control, 33(12), December 1988, 1106-1115.

20 Emami-Naeini, A., Akhter, M. M. and Rock, S. M. Robust detection, isolation and for sensor failure detection. NASA Contract Report 174825 , July 1986.

21 Merrill, W. C., De Latt, J. C. and Bruton, W. M. Advanced detection, isolation, and accommodation of sensor failure-real time evaluation. $J$. Guidance, 11(6), Nov-Dec 1988, 517-526.

22 Brown, M. and Harris, C. J Neurofuzzy adaptive modelling and control 1993, (Kluwer Press).

23 Palmer, M. T., Abbot, K. H., Schutte, P. C. and Ricks, W. R. Implementation of a research prototype onboard fault monitoring system. AIAA Paper No. 87-2777.

24 Schutte, P. C. Real-time fault monitoring for aircraft application using quantitative simulation and expert systems. AIAA Paper No. 89-3103-CP

25 Beton, R., Kingdon, J. and Upstill, C. High availability transputing systems. Proc. Transp. 1991, 2, 497-507.

26 Harris, C. J. and Fraser, R. J. C. Command and control infrastructures: the need for open systems solutions. Fourth International Conference on Advances in Communications and Control (Comcon 4), Rhodes, Greece, June 1993.

27 Harris, C. J. and White, I. Advances in command, control and communications systems: theory and applications, 1973 (Peter Peregrinus, Stevenage).

28 Graham, I. Object orientated systems. Addison Wesley, 1991.

29 Fraser, R. J. C. and Harris, C. J. Embedded command and control infrastructures for intelligent autonoms systems. DRA Protems Report ASRG 1994. 